\title{
Dirac equation description on the electronic states and magnetic properties of a square graphene quantum dot
}

\author{
Changlin Tang, ${ }^{1,2}$ Weihua Yan, ${ }^{1}$ Yisong Zheng,, ,* Guangshe $\mathrm{Li},{ }^{2}$ and Liping $\mathrm{Li}^{2}$ \\ ${ }^{1}$ Department of Physics, Jilin University, Changchun 130023, PR China \\ ${ }^{2}$ State Key Lab of Structural Chemistry, Fujian Institute of Research on the Structure of \\ Matter and Graduate School of Chinese Academy of Sciences, Fuzhou 350002, PR China
}

(Dated: May 25, 2018)

\begin{abstract}
Electronic eigen-states of a square graphene quantum dot(GQD) terminated by both zigzag and armchair edges are derived in the theoretical framework of Dirac equation. We find that the Dirac equation can determine the eigen-energy spectrum of a GQD with high accuracy even if its size is reduced to a few nanometers. More importantly, from the Dirac equation description we can readily work out the number and energy gap of the conjugate surface states, which are intimately associated with the magnetic properties of the GQD. By using the Hartree-Fock mean field approach, we study the size dependence of the magnetic ordering formation in this square GQD. We find that there exists a critical size of the width between the two zigzag edges to indicate the onset of the stable magnetic ordering. On the other hand, when such a width increases further, the magnetic ground state energy of a charge neutral GQD tends to a saturated value. These results coincide with the previous results obtained from the first principle calculation. Then, based on the Dirac equation solution about the surface state, we establish a simple two-state model which can quantitatively explain the size dependence of the magnetic ordering in the square GQD.
\end{abstract}

PACS numbers: 75.75.+a, 73.20.-r, 75.50.Xx, 75.70.Cn

\section{INTRODUCTION}

Graphene has become a subject of intense interest since the experimental success in fabricating such an atomically thin layer of graphite [1]. The valence electron dynamics in such a truly two-dimensional material is governed by a massless Dirac equation. As a result, graphene exhibits many unique electronic properties [2, 3], in comparison with the conventional semiconductor materials. From an application point of view, graphene possesses very high mobility even at room temperature [4]. Moreover, the planar geometry of graphene is of advantage to tailor various nanostructures by the current experimental means, such as the lithographic techniques [5]. So far, the obtainable graphene nanostructures include the one-dimensional(1D) nanoribbons [6] and zero-dimensional quantum dots[7]. These nanostructures are viewed as the elemental blocks to construct the graphene-based nano-devices.

Accompanying the extensive investigations on the electronic properties in bulk graphene. Graphene nanostructures also draw much attention of theoretical study [8]. First of all, some theoretical approaches to produce the effective electron confinement in graphene were proposed [9, 10, 11, 12] which is a nontrivial problem due to the Klein tunneling of the carrier in graphene 13, 14]. For example, GQD structures can be formed by patterning gates on a semiconducting graphene nanoribbon[10, 11], or by using inhomogeneous magnetic fields 12]. Then, some device applications of the graphene nanostructures were suggested, such as the spin qubits based on the coupled GQDs[10]. In addition, some electronic properties of graphene nanostructures are expected to be different from bulk graphene because of the quantum confinement and the edge effect. For instance, the spontaneous magnetization is anticipated to emerge in some graphene nanostructures 15, 16], which is attributed to the spin polarized electron occupancy at the zigzag-type edges of the nanostructures. And such a magnetic ordering has been experimentally demonstrated[17, 18]. Quite recently, the possible magnetism of graphene nanostructures with different shapes is theoretically studied in some details $19,20,21,22,23]$. For example, an infinitely long graphene nanoribbon with zigzag edges is possible to behave as a half-metallic material, in which a spin polarized current can be formed[23]. Such a property is controlled by an external electric field, which can tune the asymmetry of the band structures of the opposite spin electrons. Apart from the 1D graphene nanoribbon, GQDs with different shapes also exhibit magnetic orderings, such as square quantum dot and triangular and hexagonal quantum dots terminated by zigzag edges $19,20,21,22]$. Theoretical calculations indicate that in these zero-dimensional

${ }^{*}$ Corresponding author. Email address: zys@jlu.edu.cn 
graphene structures the magnetic ordering is so robust that can be detected at room temperature. In addition, for the square and hexagonal [20, 21] quantum dots, there is a critical size which marks the onset of the spin-polarized ground state. On the contrary, for a quantum dot with a size smaller than the critical size, the ground state is a paramagnetic state.

The aforementioned results about the magnetic properties of graphene nanostructures are obtained by means of the first-principle calculation [21] as well as the mean field approximation on a Hubbard model of a hexagonal lattice. Although these theoretical approaches can give the reliable results about the electronic properties of graphene nanostructures, their applicability is restricted within those structures with relatively small size. Furthermore, in most cases these approaches can not provide us with a clear physical picture to explain the numerical results. For example, an unambiguous explanation about the critical size for the onset of the magnetic ordering in GQDs is yet lacking. On the other hand, the Dirac equation description can just compensate for the disadvantage of the two theoretical approaches mentioned above. As a theoretical model based on the effective mass approximation, a massless Dirac equation can well describe the electron properties of the bulk graphene as well as the graphene nanostructures with relatively large sizes [24, 25]. Usually, such a model can afford analytical results, which are very helpful to explain intuitively the electronic properties associated with the relativistic quantum mechanical feature of graphene.

So far, the Dirac equation succeeds in describing the band structures of graphene nanoribbons with distinct edge types [24, 25]. In the present work, we will employ this model to study the electron states in a square GQD. With an appropriate boundary condition, we can derive an analytical solution of the electron eigenstate in such a GQD. Moreover, by a numerical calculation carried out from the mean field approximation of the Hubbard model, we investigate the size dependence of the magnetic property of the GQD. We find that there are not only the critical size, but also another characteristic size to indicate the saturation of the magnetization. Namely, it is a relatively larger size than the critical size, beyond which the spin polarization energy no longer varies with the further increase of the size of the GQD. Then, based on the analytical result obtained from the Dirac equation, we establish a simple theoretical model, which can not only reveal the physical nature of the emergence of the critical and saturated sizes, but also provide an simple way to rapidly create the quantitative result about the critical and saturated sizes, well agreeing with the numerical result of the mean field approximation.

The rest of this paper is organized as follows: Starting from the Dirac equation and using appropriate boundary conditions, the wavefunction and the dispersion relation of the electron eigenstate of the square GQD are derived in section [I] Then the eigen-energy spectra calculated from the Dirac equation and the tight binding model are compared. In section III the magnetic property of the GQD is investigated by means of Hartree-Fock mean filed theory. By establishing a two-state model, the size dependence of the magnetic ordering is quantitatively explained. Finally, the main conclusion is briefly summarized in section IV]

\section{THE ELECTRONIC STATES OF GQD}

The honeycomb lattice of the square quantum dot made of graphene monolayer is schematically shown in Fig.1. The edges of the square GQD are of two kinds, zigzag edges at the top and bottom, and armchair ones at the left and right sides. We assume that the dangling $\sigma$ bonds at the edges are passivated by hydrogen atoms. Thus, the behavior of the $\pi$ band electron near Fermi level is not nontrivially affected by the truncation of the $\sigma$ bond at the GQD edges. Within the effective mass approximation, the envelop function of the $\pi$ band electron in graphene monolayer obeys the following Dirac-like equation [26]

$$
H \psi=\gamma\left[\begin{array}{cccc}
0 & -\hat{\boldsymbol{k}}_{-} & 0 & 0 \\
-\hat{\boldsymbol{k}}_{+} & 0 & 0 & 0 \\
0 & 0 & 0 & \hat{\boldsymbol{k}}_{+} \\
0 & 0 & \hat{\boldsymbol{k}}_{-} & 0
\end{array}\right]\left[\begin{array}{c}
\phi_{A} \\
\phi_{B} \\
-\phi_{A}^{\prime} \\
-\phi_{B}^{\prime}
\end{array}\right]=E\left[\begin{array}{c}
\phi_{A} \\
\phi_{B} \\
-\phi_{A}^{\prime} \\
-\phi_{B}^{\prime}
\end{array}\right],
$$

where $\gamma=\sqrt{3} t a_{0} / 2$ with $t$ being the nearest neighbor hopping energy. In what follows we use units such that $\gamma=\hbar=1$. $\hat{\boldsymbol{k}}_{ \pm}=\hat{k}_{x} \pm i \hat{k}_{y}$ and $\hat{k}_{x(y)}=-i \partial_{x(y)}$ is an operator to measure the momentum deviation from $\boldsymbol{K}=\left(-4 \pi / 3 a_{0}, 0\right)$ or $\boldsymbol{K}^{\prime}=\left(4 \pi / 3 a_{0}, 0\right)$ point. The four components of the spinor wavefunction in Eq. (1) are associated with the total wavefunction $\Psi(\boldsymbol{r})$ by the following relationship.

$$
\psi_{\mu}\left(\boldsymbol{R}_{\mu}\right)=e^{i \boldsymbol{K} \boldsymbol{R}_{\mu}} \phi_{\mu}\left(\boldsymbol{R}_{\mu}\right)+e^{i \boldsymbol{K}^{\prime} \boldsymbol{R}_{\mu}} \phi_{\mu}^{\prime}\left(\boldsymbol{R}_{\mu}\right), \quad \mu=A, B ;
$$

and

$$
\Psi(\boldsymbol{r})=\sum_{\mu=A, B} \sum_{\boldsymbol{R}_{\mu}} \psi_{\mu}\left(\boldsymbol{R}_{\mu}\right) \xi\left(\boldsymbol{r}-\boldsymbol{R}_{\mu}\right)
$$




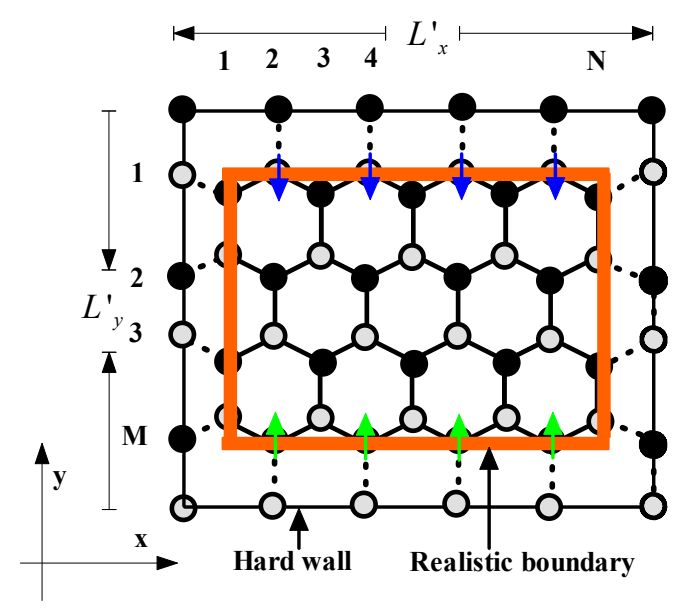

FIG. 1: A schematic of the honeycomb lattice of a square GQD terminated by both armchair and zigzag edges. There are two type of atoms $\bullet: A$ and $\circ:$ B) The realistic boundary of the GQD is denoted by the thick lines. A hard wall labeled by the peripheral rectangular framework consists of the carbon atoms just disconnected from the atoms at the edges of the GQD. The electron probability amplitudes at the atoms on the Hard wall must vanish. The size of GQD in $\mathrm{x}$ direction is denoted by $N$ or $L_{x}^{\prime}=(N+1) a_{0} / 2$, and in y direction by $M$ or $L_{y}^{\prime} \sqrt{3} a_{0}(M / 2+1 / 3)$. $a_{0}$ is the lattice constant. The upward and downward arrows at the opposite zigzag edges denote the net spin moments, which indicates the anti-ferromagnetic state. On the contrary, if the net spin moments at the two zigzag edges point at the same direction, it indicates the ferromagnetic state.

where $\xi\left(\boldsymbol{r}-\boldsymbol{R}_{\mu}\right)$ is the carbon atomic wavefunction centered at $\boldsymbol{R}_{\mu}$. And $\psi_{\mu}\left(\boldsymbol{R}_{\mu}\right)$ denotes the probability amplitude of the valence electron appearing in the vicinity of this carbon atom. For the bulk graphene, by solving Eq.(1) we can obtain the electron eigenstate which has the linear dispersion relation $\varepsilon=E / \gamma=s k$, and $s= \pm 1$ denoting the conduction and valence bands, respectively.

As for the present square GQD structure, the $\pi$ band electron obeys the same Dirac equation, but is subject to the following boundary conditions. At the zigzag edges

$$
\phi_{B}(y=0)=\phi_{B}^{\prime}(y=0)=\phi_{A}\left(y=L_{y}^{\prime}\right)=\phi_{A}^{\prime}\left(y=L_{y}^{\prime}\right)=0
$$

and at the armchair edges

$$
\phi_{\mu}(x=0)=\phi_{\mu}^{\prime}(x=0), \quad \phi_{\mu}\left(x=L_{x}^{\prime}\right)=e^{i 8 \pi L_{x}^{\prime} / 3} \phi_{\mu}^{\prime}\left(x=L_{x}^{\prime}\right) .
$$

These boundary conditions have been successfully used to work out the band structures of the graphene nanoribbons with different edges 24] and the energy spectrum of GQDs [10, 11]. They originate from the requirement that the electron probability amplitude at the hard walls around GQD must vanish. Combining Eq.(1) with Eqs.(44), we can derive the eigen solution of electron states in the square GQD. It is given by

$$
\Phi=\left[\begin{array}{c}
\phi_{A} \\
\phi_{B} \\
-\phi_{A}^{\prime} \\
-\phi_{B}^{\prime}
\end{array}\right]=C\left[\begin{array}{c}
\frac{1}{\varepsilon_{n}}\left(-p_{n} \sin (q y) e^{i p_{n} x}+q \cos (q y) e^{i p_{n} x}\right) \\
\sin (q y) e^{i p_{n} x} \\
\frac{1}{\varepsilon_{n}}\left(p_{n} \sin (q y) e^{-i p_{n} x}-q \cos (q y) e^{-i p_{n} x}\right) \\
-\sin (q y) e^{-i p_{n} x}
\end{array}\right]
$$

The corresponding eigen-energy is given by

$$
\varepsilon_{n}=s \sqrt{p_{n}^{2}+q^{2}}
$$

Although it takes the same form as the dispersion relation of the bulk graphene, in the present square GQD the wavevectors $p_{n}$ and $\mathrm{q}$ in $\mathrm{x}$ and $\mathrm{y}$ directions are both discrete. $p_{n}$ is given by

$$
p_{n}=\frac{2 n \pi}{(N+1) a_{0}}-\frac{2 \pi}{3 a_{0}}, \quad n=0, \pm 1, \pm 2 \cdots
$$

Corresponding to a given $p_{n}, q$ is determined by a transcendental equation

$$
q=p_{n} \tan q L_{y}^{\prime}
$$


By analyzing the above equation we find that the electron state with an imaginary wavevector $q=i|q|$ is allowed in the region $p_{n}>1 / L_{y}^{\prime}$, In such a state the electron wavefunction is localized in the vicinity of the zigzag edges. Accordingly, it is named as a surface state. In contrast to the surface state, we call a state with a real $q$ as the confined state. The normalization coefficient of the spinor wavefunction for the confined state is

$$
C=\left(2 L_{y}^{\prime} L_{x}^{\prime}-\sin \left(2 p_{n} L_{x}^{\prime}\right) L_{y}^{\prime} / p_{n}\right)^{-1 / 2},
$$

whereas for the surface state it is given by

$$
C=\left(\left(2 L_{x}^{\prime}-\sin \left(2 p_{n} L_{x}^{\prime}\right) / p_{n}\right)\left(\sinh \left(2 q L_{y}^{\prime}\right) / q-2 L_{y}^{\prime}\right) / 2\right)^{-1 / 2} .
$$

The eigenstate of the GQD shown in Eq.(6) can be understood in the following way. If the wavefunction is rewritten in a form $\Phi=\Phi_{K}^{p_{n}}+\Phi_{K^{\prime}}^{-p_{n}}$ with $\Phi_{K}^{p_{n}}=\left[\phi_{A}, \phi_{B}, 0,0\right]^{T}$ and $\Phi_{K^{\prime}}^{-p_{n}}=\left[0,0, \phi_{A}^{\prime}, \phi_{B}^{\prime}\right]^{T}$, we can immediately find that $\Phi_{K}^{p_{n}}$ and $\Phi_{K^{\prime}}^{-p_{n}}$ are just the wavefunctions of the eigenstates of an infinitely long zigzag nanoribbon 24] with the free wavevectors $p_{n}$ and $-p_{n}$ respectively. Therefore, the eigenstate of the GQD consists of the linear combination of the eigenstates of the zigzag nanoribbon in $\mathrm{K}$ and $\mathrm{K}$ ' valleys. The boundary condition in the armchair direction admixes $\mathrm{K}$ and $\mathrm{K}$ ' valleys. According to such an argument, we should restrict the possible surface states in the wavevector range $1 / L_{y}^{\prime}<p_{n} \leq \pi / 3 a_{0}$, where $\pi / 3 a_{0}$ is just the midpoint between $\mathrm{K}$ and $\mathrm{K}^{\prime}$ valleys in $x$ direction. When $p_{n}$ is beyond such a value, there is no longer any new surface state due to the valley admixing. Considering such a wavevector limit, we can readily determine the number of the surface states by simply counting the number of the discrete wavevectors $p_{n}$ in this range. For example, for a GQD of size $N=13$ and $M=10$ (denoted for short as N13M10), the allowed $p_{n}$ are $\pi / 21 a_{0}$ and $4 \pi / 21 a_{0}$. Therefore, the total number of the surface states in valence and conduction bands are $2 N_{t}=4$, where $N_{t}$ is the number of the allowed $p_{n}$ in the range of $1 / L_{y}^{\prime}<p_{n} \leq \pi / 3 a_{0}$. Moreover, from this method we can infer that when $N<7$ no surface state survives, independent of the value of $M$. We will see below that the surface states are responsible for the magnetic property of the GQD. Such a simple way to determine the number and energy of the surface states is helpful for us to explain intuitively the numerical result about the magnetic property of the GQD. In addition, it should be noted that in the following discussion, we only consider the GQD with odd $N$ and even $M$. Such a case corresponds to a GQD without any carbon atom at the boundary connected to the GQD by a single $\pi$ bond. Finally, although the Dirac equation can give an analytical description of the electron eigenstate in the square GQD, we have to point out that its applicability should be strictly restricted within the linear dispersion region of the $\pi$ band of graphene. It is known that the energy scope of the linear dispersion region in the $\pi$ band is about $t / 3$, away from the Dirac point. Accordingly, in the two dimensional k-space the linear dispersion region forms a circle around the $\mathrm{K}$ or $\mathrm{K}$ ' point with a radius equal to $2 /(3 \sqrt{3} a)$. Therefore, if the energy of an eigenstate of the square GQD is much lower than $t / 3$, it can be well described by the Dirac equation. In contrast, when an eigen-energy exceeds $t / 3$, the Dirac equation gets poorer to describe such an eigenstate in the square GQD. From Eq. (8) we can see that the interval between two adjacent wavevectors in the armchair direction is $\Delta p_{n}=\pi / L_{x}^{\prime}$. The discrete wavevectors in the zigzag direction should be numerically determined from Eq.(9). Therefore, it is not straightforward to find the interval between the adjacent wavevectors in this direction. However, for a relatively small $P_{n}$ we infer from Eq.(9) that such a wavevector interval is roughly equal to $\Delta q=\pi / L_{y}^{\prime}$. Thus, the two kinds of wavevector interval are inversely proportional to the sizes in the respective directions. When $\Delta p_{n}$ and $\Delta q$ become comparable to the diameter of the circle of the linear dispersion limit, there is hardly any eigenstates in the linear dispersion region. Thus, from the relation $\Delta p_{n}=\Delta q=4 /(3 \sqrt{3} a)$ we can find the minimal size of the GQD for the complete invalidity of the Dirac equation description. By a simple evaluation, we find that such a minimal size is $N_{\min }=7$ and $M_{\min }=8$. This means that the GQD with size of N7M8 is the smallest one to which the Dirac equation is applicable.

To check the validity of the Dirac equation solution about the electronic eigenstate of the GQD in some details, we compare the low-lying energy levels calculated by solving Eqs.(74-9) to the ones obtained from the tight-binding model. The tight-binding Hamiltonian of the square GQD takes a form as $H_{t b}=-t \sum_{\langle i, j\rangle} \sum_{\sigma}\left(c_{i A \sigma}^{\dagger} c_{j B \sigma}+h . c\right)$ where $c_{i \mu \sigma}^{\dagger}$ is the electron creation operator associated with a local atomic state at lattice point $i$; And $\langle i, j\rangle$ denotes any pair of the nearest neighboring carbon atoms. $\sigma=\uparrow(\downarrow)$ corresponds to the up and down spins. In the basis set consisting of the local atomic orbits, the tight-binding Hamiltonian changes into a matrix. By diagonalizing this Hamiltonian matrix we can obtain the electronic eigen-energy spectrum and the eigen wavefunctions. Noting that electronic eigen-states are spin-degenerate, though we write the spin index explicitly in the above tight-binding Hamiltonian. The electron spin becomes relevant only in the self-consistent calculation of the electron energy spectrum in the next section where the Hubbard interaction is taken into account.

The comparison of the numerical results of the low-lying eigen-energy spectra obtained by the Dirac equation as well as the tight-binding model is visualized in Fig 2. For a relatively large GQD, N35M24 as 

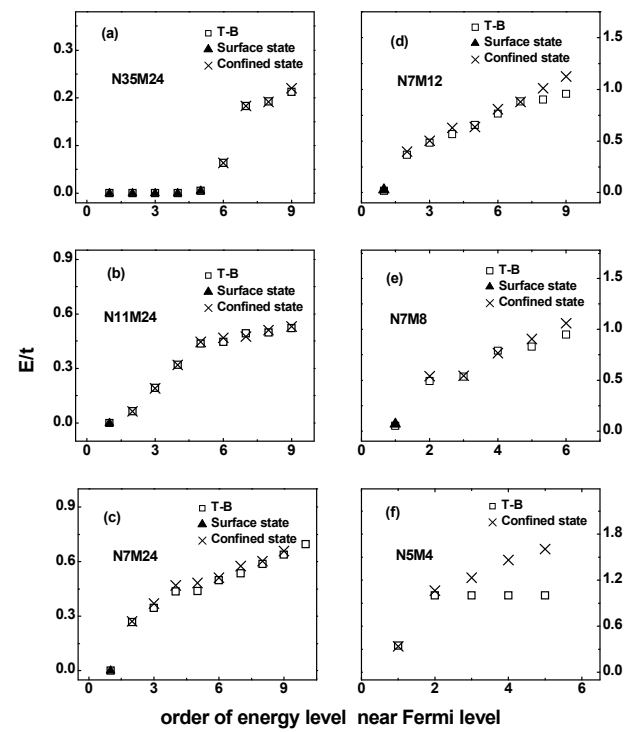

FIG. 2: A comparison of the low-lying energy levels calculated from the Dirac equation (triangle,cross) and tightbinding model (square) for GQDs with different sizes. (a) N35M24; (b) N11M24; (c) N7M24;(d) N7M12; (e) N7M8 and (f) N5M4.

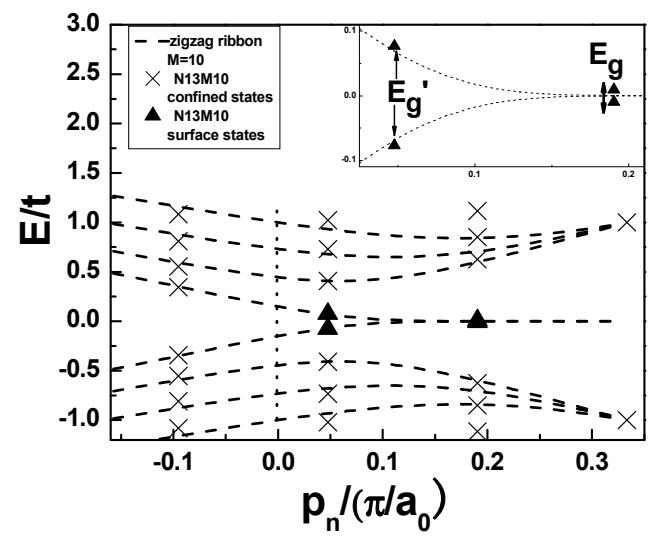

FIG. 3: Low-lying energy levels versus $p_{n}$ for a GQD of size N13M10 calculated from Dirac equation (triangle). The dashed lines depict the dispersion relation of an infinitely long zigzag nanoribbon with width $\mathrm{M}=10$, which is calculated from the tight-binding model. The inset shows more clearly the two pairs of the conjugate surface states of the GQD. And the energy gaps relative to the largest $p_{n}$ and the smallest $p_{n}$ are denoted by $E_{g}^{\prime}$ and $E_{g}$ respectively

shown in Fig[2, (a), the Dirac equation result agrees with the tight-binding result very well. Then we reduce the size of the GQD only in $\mathrm{x}$ direction. These results are plotted in Fig2(a)-(c). We can see that the Dirac equation results get poorer with the decrease of $N$. In Fig 2(c), despite $N=7$, there are a few low-lying eigen-energies obtained by the Dirac equation solution to be close to the tight-binding result. This is due to that the relatively large $M$ retains these eigenstates in the linear dispersion region. When $M$ decreases further, the results calculated by the two models deviate from each other notably, as shown in Fig 2(c)(e). The result shown in Fig/2(f) demonstrates that the Dirac equation description fails to give the correct eigen-energy spectrum for the GQD smaller than the one of N7M8. On the other hand, the result shown in Fig 2(a) indicates that at least 10 low-lying eigenstates can be safely described by Dirac equation for a GQD with size of $4 \mathrm{~nm} \times 5 \mathrm{~nm}$. In conclusion, the numerical comparison made in Fig 2 supports our simple criterion given above for the applicable limit of the Dirac equation approach to the GQD.

In Fig 3 some low-lying eigen-energies versus $p_{n}$ are plotted for a GQD of size N13M10, which is compared 
with the dispersion relation of a zigzag ribbon with width $M=10$. Although these energy levels of the GQD are discrete, from this figure we can readily infer that the energy-wavevector relation of a GQD will change into the band structure of a zigzag nanoribbon with the continued increase of the size $N$. Thus, we can say that the dispersion relation of the zigzag nanoribbon remains in the Dirac equation description of the square GQD. This is one advantage of the Dirac equation over the tight-binding model in describing the electron states of the GQD. In addition, from the Dirac equation solution, we can easily distinguish the surface states from the confined states, because the two kinds of state have distinct forms of wavefunction. This can be viewed as another advantage of the Dirac equation description. In contrast, it is difficult to identify a surface state in the tight binding model, in particular, for a GQD with small size. In fact, the Dirac equation method was previously used to describe the electron states in other GQDs 10, 11, 12. For example, for a GQD formed by applying a gate voltage on a graphene nanoribbon[10], the surface states and the confined states can also be easily distinguished from each other by using the Dirac equation method. Finally, from the inset of Fig 3 we can clearly see that corresponding to any allowed $p_{n}$, there are two conjugate surface states, belonging to the valence and conduction bands respectively. And between them there is a finite energy gap. Although there are only two pairs of surface states for the GQD shown in Fig 3 a common feature about the surface state visualized in this figure is that the pair of surface state with the minimal $p_{n}$ has just the maximal energy gap and vice versa.

\section{MAGNETIC PROPERTIES OF THE GQD}

Magnetic properties of graphene nanostructures with various shapes have drawn considerable interest. For example, some theoretical investigations indicate that a zigzag nanoribbon has a spin-polarized ground state, which is tightly associated with the surface state at the zigzag edges. This implies that a spontaneous magnetization may occur in such a nonferromagnetic material. Motivated by these previous work [15, 20, 21, 22], we now study the possible magnetic property of the square GQD. To do this, we adopt a single band Hubbard model(to incorporate the Hubbard terms into the tight binding model), and treat it within the Hartree-Fock approximation. It was previously proved that most magnetic properties of graphene nanostructure can be captured by such a simple approach [15, 16, 20]. The Hartree-Fock mean field Hamiltonian of the present GQD take a form

$$
H=H_{t b}+U \sum_{i \mu}\left(\left\langle n_{i \mu \downarrow}\right\rangle n_{i \mu \uparrow}+\left\langle n_{i \mu \uparrow}\right\rangle n_{i \mu \downarrow}-\left\langle n_{i \mu \uparrow}\right\rangle\left\langle n_{i \mu \downarrow}\right\rangle\right) .
$$

where $n_{i \mu \uparrow}\left(=c_{i \mu \uparrow}^{\dagger} c_{i \mu \uparrow}\right)$ and $\left\langle n_{i \mu \uparrow}\right\rangle$ are the electron number operator and the average electron occupation at an arbitrary lattice point respectively. Besides, $U$ is the on-site Hubbard energy. For a charge neutral GQD, the single-electron picture adopted above tells us that all eigenstates in the valence band are fully occupied, whereas all states belonging to the conduction band are empty. However, the finite Hubbard $U$ distorts such a simple electron distribution since it resists the double occupancy of a surface state by two opposite-spin electrons. As a result, spin polarized electron occupancy on individual lattice points may occur in the charge neutral GQD.

The eigen solution of the Hartree-Fock Hamiltonian for a charge neutral GQD can be obtained by iteration method. In analogy with the previous work, our iterative calculation indicates that the spin polarization situation of the obtained eigen-state of the charge neutral GQD depends on the initial spin configuration to start the iteration procedure. The different initial states will lead to the eigen-states with distinct kinds of spin polarization. At first, to begin with an initial spin configuration of Neel order, we will arrive at an eigen-state with spin polarized electron occupation on individual lattice points. In particular, on the lattice points near the two zigzag edges the spin polarization is very strong. The net spin distributions at the two zigzag edges show the anti-ferromagnetic order(AFM) in Fig.1. Second, if we start from an initial state with the uniform spin polarization at all the lattice points, the self-consistent calculation converges to an eigen-state with ferromagnetic(FM) ordering. In addition, a paramagnetic state( $\mathrm{PM})$ can be achieved if the initial spin configuration is set to be unpolarized at all lattice points. Herein we adopt the same definition about the magnetic orderings as given in the previous works [15]. The AFM state refers to that the spin moments of the carbon atoms on one zigzag edge are anti-aligned to that on the opposite edge, while the FM state means that the spin moments on both zigzag edges point at the same direction. Moreover, the PM state can be defined alike, which refers to that the spin up and down electrons are equally occupied at every lattice point. Next we focus on the size dependence of the magnetic orderings. To do this, in Fig 4 we compare the total energy difference $(\Delta E)$ between the FM(AFM) states and the PM state for the charge neutral GQD, as a function of the width $M$ between the two zigzag edges, while the width $N$ in the armchair direction takes several typical values. First of all, we can find that there exists a critical size $M_{c}$, which denotes the onset of the PM-FM(AFM) transition. Namely, only when $M>M_{c}$ is the magnetic ordering 

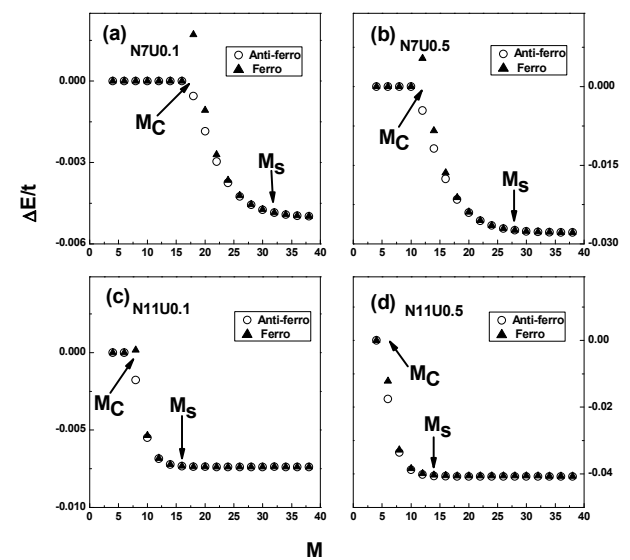

FIG. 4: Energy differences versus M between AFM(circle) and PM as well as FM(triangle) and PM states for charge neutral GQDs with different sizes and on-site energies. (a)N7U0.1t; (b)N7U0.5t; (c)N11U0.1t; and (d) N11U0.5t. The critical size $M_{c}$ and the saturated size $M_{s}$ are labeled on the curves.

stable. By comparing the results shown in Fig 4 (a-d), we find that $M_{c}$ depends on the transverse width $N$ and the Hubbard $U$ sensitively. With the increase of $N$ and $U$, the critical size $M_{c}$ decreases notably.

There is one point we have to emphasize herein about the magnetic ordering in the GQD depicted above. The FM or AFM state in the GQD considered by us and the one in an extended honeycomb lattice have distinct underlying mechanisms. It is the surface states localized at the zigzag edges to cause the AFM in the GQD. The surface states in the GQD tend to dispersionless as the width between the opposite zigzag edges getting large. Thus, a finite density of states forms in the vicinity of the Dirac point(energy zero point), which is responsible for the formation of the magnetic ordering even with a very small Hubbard $U$. However, such a surface state is absent in the extended honeycomb lattice in which there is no zigzag edge. The linear dispersion of the extended honeycomb lattice leads to the vanishing density of states at the Dirac point, which then requires a very large Hubbard U for the PM-AFM transition in such an extended lattice. In fact, a similar comparison was made in Ref 16 where the magnetic ordering in a zigzag ribbon only needs a much smaller(infinitely small, in fact) Hubbard U than in the extended honeycomb lattice. In analogy with the GQD, the zigzag ribbon possesses surface states which form a flat band at the Dirac point, leading to the magnetic ordering even with an infinitely small U. In short, we think the magnetic ordering formed by a small $U$ is due to the existence of the surface state in the GQD. The spin polarized electron occupancy is notable only in the regions near the zigzag edges. The value of the Hubbard $U$ we used in our work is too small to show the formation of the AFM state following the mechanism of the extended lattice(from the result shown in Ref 16 we can find out that such a critical U is larger than $2 \mathrm{t}$ ).

Another noticeable feature in Fig 4 is that the energy difference tends to a saturated value when the width $M$ exceeds a specific value $M_{s}$. Hereafter we call $M_{s}$ the saturated size. It is quantitatively determined following such a way: when the size of the GQD increases from $M_{s}$ to $M_{s}+2$, the relative increment of $\Delta E$ should be less than 1\%. In addition, from Fig 4 we can find that the energy of the AFM state is notably lower than that of the FM state in the region $M_{c}<M<M_{s}$. This indicates that the AFM state in the region between the critical size and the saturated size is just the ground state of the charge neutral GQD [21, 27]. On the other hand, when $M$ is sufficiently large, the energy difference between the FM and AFM states becomes indistinguishable. This can be explained in such a way: The surface states in the GQD can be viewed as the bonding or anti-bonding states arising from the interaction between two kinds of surface states located at the opposite zigzag edges. In fact, each of such two surface states belongs to a semi-infinite two-dimensional graphene terminated by a zigzag edge. When $M$ is very large, the interaction between the two kinds of surface states gets weak. As a result, the exchange integral between them which determines the relative orientations of the net spin moments at two zigzag edges becomes negligibly small. Thus the energy difference between FM and AMF states tends to zero. Finally, we have to point out that the existence of the critical size for a hexagonal and square graphene quantum dots was previously reported 20, 21, 22], based on the first principle calculation. The result about the square GQD agrees quantitatively with our present result obtained by the mean field approximation 21, 22]. However, a clear explanation about the critical size is yet lacking.

Next we try to give a reasonable explanation about the occurrence of the critical and saturated sizes. 
At the first step, we discuss the relation between the magnetic order formation and the electron occupancy on the surface states. Accordingly, we can work out a simple criterion which can qualitatively explain the numerical result about the critical size obtained above by the Hartree-Fock mean field theory. Then, we establish a two-state model which can give a quantitative explanation to the numerical result about the critical and saturated sizes. All these arguments benefit from the Dirac equation solution about the singleparticle surface states. As analyzed in the preceding section, the number of the surface states in the range $1 / L_{y}^{\prime}<p_{n} \leq \pi / 3 a_{0}$ is finite. Corresponding to a specific $p_{n}$, there are two conjugate surface states, belonging to the conduction and valence bands respectively. And the energy gap between them depends on $p_{n}$ and the size of the GQD. We consider specially the two conjugate surface states with the maximal $p_{n}$. In comparison with other surface states, this pair of surface states has the smallest energy gap. The finite Hubbard $U$ alters the single electron energy spectrum since it affords an on-site Coulomb repulsive potential. For example, in a presumed paramagnetic state, the surface state with a specific spin $\sigma$ in the valence band will rise by $U\left\langle n_{i \bar{\sigma}}\right\rangle=U / 2$ due to the Coulomb repulsion. When such a shift makes the surface state in the valence band is aligned with the surface state in the conduction band, the system is likely to show the spontaneous magnetization. Following such an analysis, we obtain a simple criterion to determine the critical size. $M_{c}$ is the size at which the inequality

$$
E_{g}<U / 2
$$

begins to hold true, where $E_{g}$ is the energy gap between the pair of surface states corresponding to the maximal $p_{n}$. By calculating $E_{g}$ we can obtain the critical size $M_{c}$. Using such a simple criterion, we estimate the critical size $M_{c}$, varying with the size $N$ as well as the Hubbard $U$. These results are shown in Fig.5. In comparison with the Hartree-Fock mean field result, we find that the criterion given by Eq. (13) can roughly account for the dependence of the critical size on $N$ as well as $U$.

Although the above criterion is not too bad, we will show that a more quantitative explanation about the critical size is available. Now that the onset of the magnetic ordering is controlled by the pair of surface states with the minimal energy gap, we establish a simple two-state model by retaining only this pair of surface states in the mean field Hamiltonian. Thus, the Hartree-Fock Hamiltonian for this two-state model takes a form as

$$
H^{t s}=\sum_{s \sigma} \varepsilon_{s} c_{s \sigma}^{\dagger} c_{s \sigma}+U \sum_{i \mu \sigma}\left\langle\hat{m}_{i \mu \sigma}\right\rangle \hat{m}_{i \mu \bar{\sigma}} .
$$

here $s= \pm$ denotes the two surface states belong to the conduction and valence bands respectively. $c_{s \sigma}\left(c_{s \sigma}^{\dagger}\right)$ is the electron annihilation(creation) operator of the surface state of quantum index $s \sigma$. The electron number operator $\hat{m}_{i \mu \sigma}$ counts only the contributions of the two surface states to the electron occupancy on the individual carbon atoms. According to such a meaning, it is associated with the electron number operator of the two surface states via a relation

$$
\hat{m}_{i \mu \sigma}=\sum_{s}\left|\psi_{\mu}^{s}(i)\right|^{2} \Omega c_{s \sigma}^{\dagger} c_{s \sigma},
$$

here $\Omega=\sqrt{3} a_{0}^{2} / 2$ is the area of unit cell, and $\left|\boldsymbol{\psi}_{\mu}^{s}(i)\right|^{2}$ is just the probability of the electron in surface state (s) appearing in the vicinity of the carbon atom $\mu$ at lattice point $i$, which can be directly calculated from the analytical wavefunction in Eq.(2). For the charge neutral GQD, the two surface states accommodate two electrons with opposite spins. We thus have $\sum_{s \sigma} c_{s \sigma}^{\dagger} c_{s \sigma}=2$. In addition, it should be noted that in such a two-state model we have ignored the contributions to the average electron occupancy on the individual lattice points from other occupied single electron eigen-states, except for the two surface states retained in this model. This is because that other occupied electron states in the valence band only provide a spinunpolarized charge background, which influences the opposite spin electrons in the two surface states on an equal footing. Substituting Eq.(15) into Eq.(14), we obtain the following diagonal Hamiltonian

$$
H^{t s}=\sum_{s, \sigma}\left(\varepsilon_{s}+\sum_{s^{\prime}} U_{0}\left\langle c_{s^{\prime} \bar{\sigma}}^{\dagger} c_{s^{\prime} \bar{\sigma}}\right\rangle\right) c_{s \sigma}^{\dagger} c_{s \sigma},\left(s^{\prime}= \pm s\right)
$$

with

$$
U_{0}=\sum_{\mu} U \cdot \Omega \int d r\left|\psi_{\mu}^{s}(\boldsymbol{r})\right|^{2} \cdot\left|\psi_{\mu}^{s^{\prime}}(\boldsymbol{r})\right|^{2} .
$$

Here $U_{0}$ can be calculated analytically by the Dirac equation wavefunction given in Eq.(2), or numerically by tight-binding model instead. By a simple derivation from the Dirac equation wavefunction we obtain the 
analytical form about $U_{0}$. It is given by

$$
U_{0}=\frac{3 \sqrt{3} U\left(\frac{\sinh 4 q L_{y}}{16 q}-\frac{\sinh 2 q L_{y}}{2 q}+\frac{3 L_{y}}{4}\right)}{L_{x}\left(\frac{\sinh 2 q L_{y}}{q}-2 L_{y}\right)^{2}}
$$

For the PM state, two electrons occupy the valence band surface state $\left(\varepsilon_{-}\right)$with opposite spins. From the diagonal Hamiltonian given above, we can immediately obtain that the energy of the two electrons is equal to $E_{p m}=2 \varepsilon_{-}+2 U_{0}$. On the other hand, for the possible magnetic ordering state, the two electrons occupy the two distinct suface states with the same spin. The correspond energy is then $E_{m o}=\varepsilon_{-}+\varepsilon_{+}$. The critical size for the magnetic ordering to become stable corresponds to $E_{m o} \leq E_{p m}$, Namely,

$$
E_{g} \leq 2 U_{0} .
$$

By means of this criterion we can determine the critical size $M_{c}$. It should be noted that although the two-state model is established according to the Dirac equation description of the surface states, the two parameters $E_{g}$ and $U_{0}$ can also be calculated from the tight-binding model. Therefore, the two-state model is expected to be still valid even when the conjugate surface states are beyond the linear dispersion region. The critical size obtained from this two-state model is shown in Fig 5 In comparison with the mean field result, we find that the two-state model can give a quantitative explanation about the critical size, as a function of $U$ and $N$. Besides, in Fig 5 (a) we also find that the two-state model with the parameters evaluated from the Dirac equation can no longer predict the critical size satisfactorily with the increase of $N$. This can be readily understood. In the two-state model, we consider the pair of surface states with the maximal $p_{n}$, which gets away from the center of the valley with the increase of $N$. As a result, the Dirac equation becomes poorer to give a quantitative description about the electron probability amplitude. Instead of the Dirac equation solution, if we evaluate the parameters $E_{g}$ and $U_{0}$ from the tight binding model, as shown in Fig 5 the two-state model always gives a satisfactory result, which demonstrates that the two-state model has captured the main mechanism dominating the spin polarization in the GQD. Finally, we would like to point out that our numerical result about the critical size shown in Fig 5 coincides with those obtained by the first principle calculation or the mean field method in the previous work 21, 22, 28]. In particular, our calculation indicates that when $N<7$ no surface state exists, hence no magnetic ordering occurs. And when $N=7$ the critical size is $M_{c}=8$. These quantitative results were also produced in the relevant work obtained by the first principle calculation[29]. According to solution about the surface state given in the previous section, we can predict that the critical size $M_{c}$ will tend to zero, as the size $\mathrm{N}$ becomes sufficiently large. This is because that there must be a dispersionless surface state pair when $\mathrm{N}$ becomes sufficiently large, regardless of the size M. Thus, The GQD becomes an infinitely long zigzag ribbon which always possesses the dispersionless surface states. As a result, the spontaneous magnetization can occur at an arbitrarily small $\mathrm{M}$.

Now we turn to discuss the occurrance of the saturated size $M_{s}$, based on the Dirac equation description of the surface states. The mean field result of $M_{s}$ as a function of $N$ is shown in Fig 6 , It depends on $N$ nonmonotonously, in contrast to its insensitive dependence on the Hubbard $U$. For example, when $N$ increases from 7 to $11, M_{s}$ decreases notably, followed by an abrupt rise at $N=13$. Such a situation recurs when $N$ increases further. According to our solution about the surface state, when $N$ increases within a small range, such as from $N=7$ to 11, the number of surface states does not change. The pair of surface states with the minimal $p_{n}$ has the maximal energy gap(we denoted it as $E_{g}^{\prime}$ in the inset of Fig 3). According to our two-state model, such a gap goes against the formation of spin polarization in the two conjugate surface states. And the energy difference between the spin polarized and unpolarized electron occupancies on the two surface states is equal to $E_{g}^{\prime}-2 U_{0}$. From Eqs.(74) we infer that with the increase of $M, q$ goes close to $p_{n}$. As a result, $E_{g}^{\prime}$ tends to vanish. Meanwhile, as q goes close to $p_{n}, U_{0}$ tends towards a constant, because the wavefunction (Eq 6 ) of surface states near zigzag edges tends towards a constant value. When $E_{g}^{\prime}$ becomes sufficiently small, such a pair of surface states, hence all pairs of surface states, have stable contribution to the spin polarization. Thus, the total energy difference shown in Fig 4 tends to a saturated value. In comparison to the case of $N=7$, the GQD with $N=11$ has a smaller $E_{g}^{\prime}$ corresponding to the same $M$. Thus the GQD with $N=11$

corresponds to a smaller $M_{s}$. However, when $N$ increases further, a new surface state comes into being, with an larger $E_{g}^{\prime}$ than the case of $N=7$. Thus a larger $M_{s}$ is needed. This justifies the abrupt rise of $M_{s}$ at $N=13$ as shown in Fig.6. In such a spirit, we can establish a simple criterion by which we can estimate the value of $M_{s}$. This is

$$
p_{n}-q \leq \eta .
$$

where $p_{n}$ is the smallest wavevector in the surface state allowed region. $\eta$ is an appropriate small quantity to characterize the extent that q approaches to $p_{n}$. The energy gap $E_{g}^{\prime}$ is then sufficiently small and $U_{0}$ 

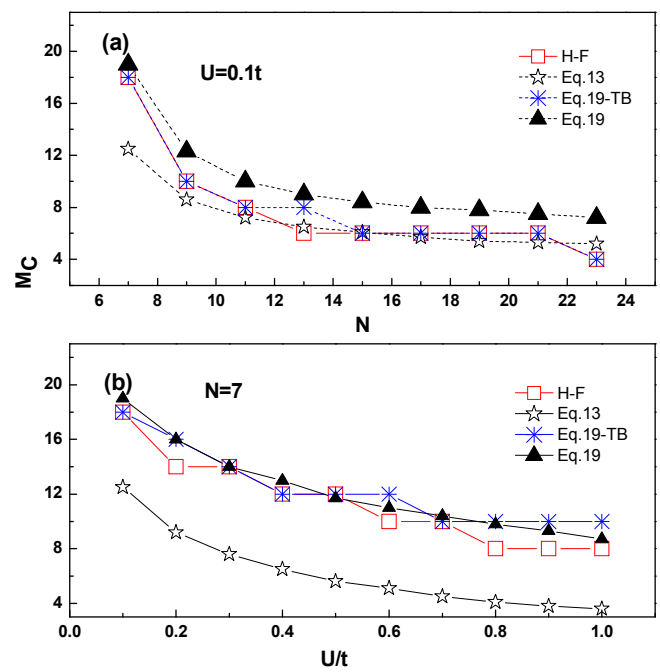

FIG. 5: (a) The critical size $M_{c}$ versus the transverse size $N$. (b)The critical size $M_{c}$ versus $U$. The square symbol denotes the Hartree-Fock mean field result; The star symbol is the result obtained from the simple criterion given by Eq.(13). The triangle and cross symbols for the results obtained from the two-state model. The former is the result that the parameters $E_{g}$ and $U_{0}$ are evaluated from Dirac equation. And the latter is the result with $E_{g}$ and $U_{0}$ calculated from tight binding model.

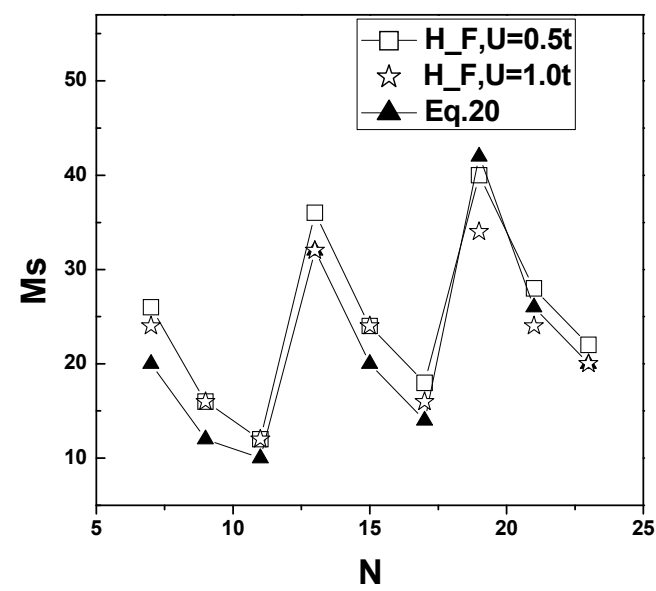

FIG. 6: The saturated size $M_{s}$ versus the transverse size $N$. The Hartree-Fock mean field results for $\mathrm{U}=0.5 \mathrm{t}(\mathrm{square})$ and $\mathrm{U}=\mathrm{t}(\mathrm{star})$ as well as the estimated result by using Eq.20 (triangle) are compared. These results coincide with each other very well.

becomes a constant. For the numerical calculation, we choose $\eta=10^{-4} / a_{0}$. From Fig 6 we can find that such a simple rule creates a saturated size $M_{s}$ which agrees with the Hartree-Fock mean field result very well. Finally, we have to point out that the result in Fig 6 only shows the saturated size $M_{s}$ in a very finite range of the size $\mathrm{N}$, because that the self-consistent calculation becomes rather time-consuming as $\mathrm{N}$ gets larger. But we can predict that as $\mathrm{N}$ increases furthermore, the oscillation of the $M_{s}$ will become weak since the discrete wavevector $P_{n}$ tends to a continuous quantity. Finally the saturated size $M_{s}$ tends to that of the infinitely long zigzag ribbon. 


\section{CONCLUSION AND REMARKS}

The electronic eigenstates of a square GQD terminated by both zigzag and armchair edges have been studied analytically in the theoretical framework of Dirac equation. By comparing with the result of tight binding model, we find that the Dirac equation can well describe the electron eigen-states even if the size of a GQD reduces to a few nanometers. Moreover, the Dirac equation method has advantages over the tight binding model in two aspects. At first, the Dirac equation solution about the electron eigen-states can tell us not only the energy levels but also the dispersion relation. Then, from the Dirac equation solution, we can readily determine the number of the surface states. In addition, by using the Hartree-Fock mean field theory, we have also investigated the magnetic properties of the square GQD. We find that stable magnetic ordering states are allowed for a charge neutral GQD with an appropriate size. The magnetic ordering depends on the width between two zigzag edges sensitively. Only when the width is larger than a critical size is the magnetic ordering stable. On the other hand, when this width becomes sufficiently large, the magnetic ordering ground state energy tends towards a saturated value. We find that the critical size is dominated by the pair of surface states with the minimal energy gap, while the saturated size is determined by the pair of surface states with the maximal energy gap. Based on the Dirac equation description, we establish a simple model in which only the two dominated surface states are incorporated. Consequently, this two-state model can quantitatively explain the size dependence of the magnetic ordering of the square GQD. Thus, by virtue of such a toy model, we can estimate rapidly the characteristic sizes for the formation of magnetic ordering in the GQD.

\section{Acknowledgments}

This work is supported by the National Natural Science Foundation of China under Grants No. 10774055 and No. 20771101, also supported by a grant from Hundreds Youth Talents Program of CAS (Li GS)

[1] K. S. Novoselov, A. K. Geim, S. V. Morozov, D. Jiang, Y. Zhang, S. V. Dubonos, I. V. Grigorieva, and A. A. Firsov, Science 306, 666 (2004).

[2] J. C. Meyer, A. K. Geim, M. I. Katsnelson, K. S. Novoselov, T. J. Booth, and S. Roth, Nature 446 , 60 (2007).

[3] Y. Zheng and T. Ando, Phys. Rev. B 65, 245420 (2002).

[4] K. S. Novoselov, A. K. Geim, S. V. Morozov, D. Jiang, Y. Zhang, M. I. Katsnelson, I. V. Grigorieva, and S. V. Dubonos, nature 438, 197 (2005).

[5] Y. Zhang, Y. W. Tan, H. L. Stormer, and P. Kim, Nature 438, 201 (2005).

[6] M. Y. Han, B. Ozyilmaz, Y. Zhang, and P. Kim Phys. Rev. Lett. 98, 206805 (2007).

[7] A. Geim and K. Novoselov, Nature Materials 6,183 (2007).

[8] A. H. Castro Neto, F. Guinea, N. M. R. Peres, K. S. Novoselov and A. K. Geim, 0709.1163v1[cond.mat.other]

[9] J. Nilsson, A. H. Castro Neto, F. Guinea, and N. M. R. Peres, Phys. Rev. B 76, 165416 (2007).

[10] B.Trauzettel, DV. Bulaev, D. Loss, and G. Burkard, Nature Physics 3, 192 (2007)

[11] P. G. Silvestrov and K. B. Efetov, Phys. Rev. Lett. 98, 016802 (2007).

[12] A. De Martino, L. Dell'Anna, and R. Egger, Phys. Rev. Lett. 98, 066802 (2007).

[13] V. V. Cheianov and V. I. Fal'ko, Phys. Rev. B 74, 041403R (2006).

[14] M. I. Katsnelson, K. S. Novoselov, and A. K. Geim, Nature Phys. 2, 620 (2006).

[15] L. Pisani, J. A. Chan, B. Montanari, and N. M. Harrison, Phys. Rev. B 75, 064418(2007).

[16] M. Fujita, K, Wakabayashi and K. Nakada, J. phys. Soc. Japan 65,1920 (1996)

[17] Y. Shibayama, H. Sato, T. Enoki, and M. Endo, Phys. Rev. Lett. 84, 1744 (2000).

[18] T. Enoki and Y. Kobayashi, J. Mater. Chem. 15, 3999 (2005).

[19] Motohiko Ezawa, Phys. Rev. B 76, 245415 (2007).

[20] J. Fernandez-Rossier and J. J. Palacios, Phys. Rev. Lett. 99, 177204 (2007).

[21] De-en Jianga, Bobby G. Sumpter and Sheng Dai, J. chem. phys. 127 , 124703 (2007).

[22] Oded Hod, Veronica Barone, and Gustavo E. Scuseria, Phys. Rev. B 77, 035411 (2008).

[23] Young-Woo Son, Marvin L. Cohen and Steven G. Louie, nature 444, 347 (2006).

[24] L. Brey and H. A. Fertig, Phys. Rev. B 73, 235411 (2006)

[25] L. Brey and H. A. Fertig, Phys. Rev. B 75, 125434 (2007)

[26] T. Ando and T. Nakanishi,J. Phys. soc. Japan. 67, 1704 (1998).

[27] E. H. Lieb, Phys. Rev. Lett. 62, 1201 (1989)

[28] Hideki Kumazaki and Dai S. Hirashima, J. Phys. Soc. Jpn. 77, 044705 (2008)

[29] In Ref 22 it was stressed that at least three consecutive units along the zigzag edges is required to present spin polarization in the GQD. This agrees exactly to that $N=7$ is the minimal size for the occurance of the magnetic ordering. In table 1 of Ref 21 , it was shown that a GQD of size of $(X=3, Y=3)$ dose not present any magnetic 
ordering, which corresponds to the size of $N=7$ and $M=4$ by our definition. Moreover, when $X=3$ and $Y=5$ (equivalent to $N=7$ and $M_{c}=6$ ) the magnetic ordering begins to appear, which is slightly smaller than our result for the critical size $\left(M_{c}=8\right.$ for $N=7$, see Fig.4(b)). But it should be noticed that the Hubbard $\mathrm{U}(\approx 2 t)[15]$ in Ref 21 is larger than that used by us $(\mathrm{U}=\mathrm{t})$. Of course, a larger $\mathrm{U}$ corresponds to a smaller critical size. If we extrapolate our result shown in Fig.4(b) to $\mathrm{U}=2 \mathrm{t}$, the critical size is expected to be close to $M_{c}=6$. 